\title{
On the distribution of urine output in normally cycling women
}

\author{
Delwyn G. Cooke ${ }^{1}$, Leonard F. Blackwell ${ }^{2}$, Simon Brown ${ }^{3,4}$ \\ ${ }^{1}$ Science Haven Limited, Palmerston North, New Zealand, e-mail: D.G.Cooke@ massey.ac.nz \\ ${ }^{2}$ Institute of Fundamental Sciences, Massey University, Palmerston North, New Zealand, \\ e-mail: L.F.Blackwell@ massey.ac.nz \\ ${ }^{3}$ Deviot Institute, Deviot, Tasmania 7275, Australia, e-mail: Simon.Brown@deviotinstitute.org \\ ${ }^{4}$ College of Public Health, Medical and Veterinary Sciences, James Cook University, \\ Queensland 4811, Australia
}

\section{SUMMARY}

\begin{abstract}
It has been suggested that it is possible to monitor the menstrual cycle by measuring the concentration of urinary reproductive steroids. This neglects the variation in void volume and in urine production rate. In neither case has any systematic analysis been reported previously. Overnight urine samples were collected each day for one complete cycle by 24 women and the void volumes and intervoid times were recorded. The void volume and urine production rate were approximately lognormally distributed and the intervoid time was approximately normally distributed. Using these distributions we consider the implications of the variation in void volume and urine production rate for the comparison of the concentrations of a urinary analyte in two samples.
\end{abstract}

Key words: distribution, menstrual cycle, urine, urine production rate

\section{Introduction}

It has long been standard practice to assay urine analytes in samples collected over $24 \mathrm{~h}$ (Thudichum, 1858). However, $24 \mathrm{~h}$ urines are difficult to obtain reliably, especially from non-hospitalised subjects (Miller and Simundic, 2013), and their usefulness has been debated frequently (Gaspari et al., 2006; Shidham and Herbert, 2006; Côté et al., 2008; Ji et al., 2012). This has led to the use of overnight and, sometimes, 'spot' urine samples instead. Overnight urine samples, in particular, have been shown to be useful in monitoring the menstrual cycle, providing that the time between voids is at least $3 \mathrm{~h}$ (Brown, 2011), as is 
recommended in the collection of 'spot' samples for other purposes (Shidham and Herbert, 2006).

Irrespective of the sampling regime, the void volume and, often, the time between voids vary, so it is necessary to account for the resulting changes in quantitative assays of any urinary analyte (Brown et al., 2018). The natural approach wherever high quality data are required is simply to measure the urine production rate, as has routinely been done by users of the Ovarian Monitor (Brown et al., 1989; Blackwell et al., 2003; Blackwell et al., 2012; Blackwell et al., 2013; Blackwell et al., 2016). The analyte excretion rate is then calculated by multiplying the analyte concentration by the urine production rate (Brown et al., 2017). Others have used urinary creatinine to approximate this correction, based on the rather questionable notion that the rate of creatinine excretion is constant (Perrone et al., 1992; Remer et al., 2002; Barr et al., 2005), so that changes in concentration are inversely proportional to changes in urine production rate (which means that creatinine correction involves dividing the analyte concentration by the creatinine concentration to obtain a quantity that is related to the analyte excretion rate). Creatinine correction is in widespread use in the estimation of urinary analytes and it is not uncommon that creatinine correction is also applied to $24 \mathrm{~h}$ urines (Lane et al., 2006) or that the 'accuracy' or 'completeness' of a $24 \mathrm{~h}$ urine sample is assessed using creatinine (Côté et al., 2008). Other NMR-specific means of correcting for urine volume have been proposed that are equivalent to dividing an analyte concentration by a combination of analyte concentrations (Craig et al., 2006; Dieterle et al., 2006; Rasmussen et al., 2011), and these tend to be used in those contexts in preference to the physiologically relevant urine production rate correction.

More recently, it has been suggested that any direct urine production rate correction could be avoided in monitoring the menstrual cycle by using deviations from the smoothed, logarithmically transformed concentrations of one analyte (pregnanediol 3-glucuronide or PdG in this case) to correct concentration estimates of another analyte (oestrone 3-glucuronide or E1G) (Miro et al., 2004; Ziomkiewicz et al., 2012). Most recently, it has been suggested that it is not necessary to correct for urine production rate at all (MacGregor et al., 2006; Ecochard et al., 2013; Johnson et al., 2015). Notably, Johnson et al. (2015) appear 
to consider that their approach is quantitative despite the complete absence of any consideration of the inevitable variation in void volume. In fact they report what they describe as 'normal ranges' for the concentration of each of several urinary analytes, including E1G and PdG. Without any consideration of the implications, these authors have confused a quasi-random number (the analyte concentration measured in units of $\mathrm{mol} \mathrm{L}^{-1}$ or $\mathrm{g} \mathrm{L}^{-1}$ ) for a number that reflects both the analyte concentration and the void volume (the excretion rate measured in $\mathrm{mol} \mathrm{h}^{-1} \mathrm{or} \mathrm{g} \mathrm{h}^{-}$ $\left.{ }^{1}\right)$ that is the physiologically relevant variable (Brown et al., 2018). Where the analyte concentration, rather than the analyte excretion rate, is of interest, as may be the case in urine metabolomics, it might be satisfactory not to correct for the urine production rate; but in circumstances where the analytes are used to monitor physiological processes, such as the menstrual cycle, it is unlikely to be sufficient.

We have reported previously that the urine production rate of a small number of samples is approximately lognormally distributed (Brown et al., 2018), but it is not clear what the distribution of the void volume and intervoid time might be. Moreover we have not considered the implications of the distribution for the estimation of urinary analyte concentrations. Here, we address several questions. First, how variable is the overnight urine production rate? What is the range and how much day-to-day variation is there? Second, given the likely impact on analyte concentration (Brown et al., 2018), how large and how variable (range and day-to-day) are the void volumes? Third, are the collection times for these samples consistent with larger scale studies of the duration of sleep (on the assumption that these might approximate the overnight collection interval)? Finally, what is the significance of void volume variation for the estimation of urinary analytes?

\section{Materials and methods}

\subsection{Collection of overnight urine samples}

Each of 24 normally cycling women from Palmerston North collected daily urine samples for one complete cycle as described previously (Cooke, 2000). This yielded a total of 653 urine samples, for each of which the void volume and the time since the previous void (the intervoid time) were recorded and the urine 
production rate (often called the 'urine volume') was calculated from these. In all cases, the intervoid time was at least $3 \mathrm{~h}$.

\subsection{Statistical analysis}

Distributions were fitted by maximum likelihood and the most suitable of those considered (normal, lognormal, log-logistic, gamma and inverted beta) was selected based on the Akaike information criterion as described previously (Brown, 2017). Confidence intervals for the correlation coefficient, median, skewness and kurtosis excess were calculated using 1000 bootstrap replicates. All analyses were carried out in R (Ihaka and Gentleman, 1996).

\section{Results}

\subsection{Overall distributions}

The void volumes ranged from $50 \mathrm{~mL}$ to $980 \mathrm{~mL}$ around a median of $370 \mathrm{~mL}$ (Table 1). However, the distribution of the void volumes was positively skewed (Table 1), consistent with it being approximately lognormal (Figure 1, A and B), such that $25 \%$ of the samples were in the range of $500 \mathrm{~mL}$ to $980 \mathrm{~mL}(1.4-2.6$ times the median) and $25 \%$ of the samples were in the range of $50 \mathrm{~mL}$ to $250 \mathrm{~mL}$ (0.14-0.67 times the median). The median time between voids was $8.67 \mathrm{~h}$ and ranged from the prescribed minimum time $(3 \mathrm{~h})$ to about $14 \mathrm{~h}$ (Table 1). The distribution of intervoid times was not significantly skewed (Table 1), consistent with an approximately Gaussian distribution in which 50\% of times were between $7.75 \mathrm{~h}$ and $9.5 \mathrm{~h}$ (Figure 1, C and D). The median of the urine production rate was about $43 \mathrm{~mL} \mathrm{~h}^{-1}$ and ranged from $10 \mathrm{~mL} \mathrm{~h}^{-1}$ to $187 \mathrm{~mL} \mathrm{~h}^{-1}$ (Table 1), so that the maximum was 18 times the minimum. The distribution was positively skewed (Table 1) consistent with it being approximately lognormal (Figure 1, E and F). While $50 \%$ of the samples were reasonably consistent $\left(31-59 \mathrm{~mL} \mathrm{~h}^{-1}\right.$ or 0.73 1.39 times the median), $25 \%$ of samples varied much more $\left(59-187 \mathrm{~mL} \mathrm{~h}^{-1}\right.$ or $1.45-4.4$ times the median) and $25 \%$ of samples were small (0.24-0.73 times the median). 
Table 1. Summary of the properties of the distributions of void volume, intervoid time and urine production rate (Figure 1), based on $n=653$ samples from 24 women. Where specified, $95 \%$ confidence intervals $(95 \% \mathrm{CI})$ were estimated from 1000 bootstrap replicates

\begin{tabular}{llll}
\hline & Void volume $(\mathrm{mL})$ & Intervoid time $(\mathrm{h})$ & $\begin{array}{l}\text { Urine production } \\
\text { rate }(\mathrm{mL} / \mathrm{h})\end{array}$ \\
\hline mean $( \pm \mathrm{SD})$ & $398 \pm 174$ & $8.6 \pm 1.6$ & $48 \pm 24$ \\
median [95\% CI] & $370[350,375]$ & $8.67[8.50,8.75]$ & $43[40,44]$ \\
skewness $^{*}[95 \% \mathrm{CI}]$ & $0.71[0.57,0.86]$ & $-0.21[-0.44,0.05]$ & $1.45[1.16,2.03]$ \\
kurtosis excess* $[95 \% \mathrm{CI}]$ & $0.1[-0.2,0.6]$ & $0.9[0.5,1.5]$ & $3.1[1.5,6.9]$ \\
range & {$[50,980]$} & {$[3.00,14.08]$} & {$[10,186]$} \\
\hline
\end{tabular}

${ }^{*}$ The skewness and the kurtosis excess of a normally distributed variable are zero.
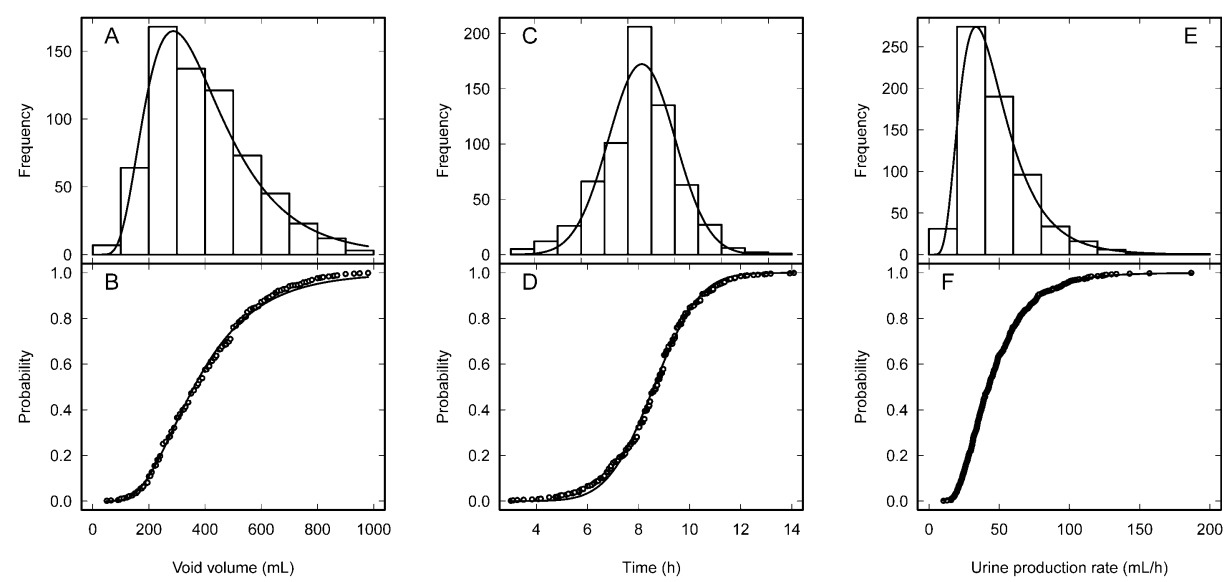

Figure 1. Distribution of void volume (A and B), intervoid time (C and D) and urine production rate ( $\mathrm{E}$ and $\mathrm{F}$ ) for $n=653$ overnight samples from 24 different women. In each panel the curve is the appropriate form of the lognormal distribution fitted by maximum likelihood (the details are given in Table 1)

Overall, the correlation between the intervoid time and the void volume was weak $(r=0.10)$ and that between the intervoid time and the urine production rate was slightly stronger $(r=-0.33)$. However, the latter is inevitable given that the variables are a ratio and its denominator (Pearson, 1897). Taking each subject individually did not alter these significantly. The median correlation between the intervoid time and the void volume was $r=0.16(95 \% \mathrm{CI}=[0.07,0.24])$ and that between the intervoid time and the urine production rate was $r=-0.19(95 \% \mathrm{CI}$ $=[-0.37,-0.13])$. 


\subsection{Day-to-day variation}

The day-to-day variation in void volume, intervoid time and the urine production rate was approximately normally distributed (Figure 2), as expected (Dudewicz and Mishra, 1988 p. 267; Lo, 2012). In each case the skewness was not significantly different from zero, but the kurtosis excess was significantly different from zero (Table 1), indicating that the weight of the tails of the distribution was slightly greater than would be expected for a normally distributed variable (for which the kurtosis excess is zero). The range of variation in the daily change in the void volume was $-645 \mathrm{~mL}$ to $605 \mathrm{~mL}$ (interquartile range $(\mathrm{IQR})=[-120,120] \mathrm{mL})$, so that for $25 \%$ of days successive void volumes differed by between $220 \mathrm{~mL}$ and $645 \mathrm{~mL}$. The day-to-day range of variation of the urine production rate was $-136 \mathrm{~mL} \mathrm{~h}^{-1}$ to $115 \mathrm{~mL} \mathrm{~h}^{-1}(\mathrm{IQR}=[-13.6,13.9] \mathrm{mL}$ $\mathrm{h}^{-1}$, Table 2), so that for $25 \%$ of days successive urine production rates differed by between $28.5 \mathrm{~mL} \mathrm{~h}^{-1}$ and $135.6 \mathrm{~mL} \mathrm{~h}^{-1}$.
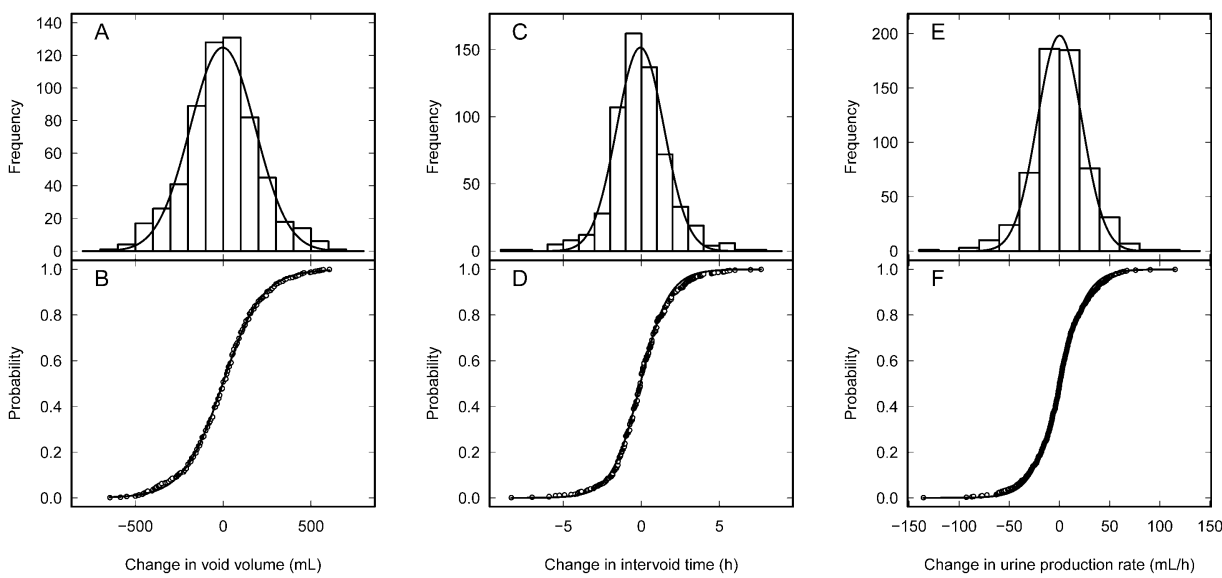

Figure 2. Distribution of the day-to-day change in void volume (A and B), intervoid time $(\mathrm{C}$ and $\mathrm{D})$ and urine production rate $(\mathrm{E}$ and $\mathrm{F})$ for $n=653$ samples from 24 different women. The change for the $i$ th cycle in the variable $(x)$ was calculated each day $\left(j=2,3, \ldots, m_{i}\right)$ of the cycle as $x_{i j}-x_{i(j-1)}$. In each panel the solid curve is a normal distribution fitted to the data by maximum likelihood (the details are given in Table 2) 
Table 2. Summary of the properties of the distributions of the day-to-day change in void volume, intervoid time and urine production rate (Figure 2), based on $n=653$ samples from 24 women. Where specified, 95\% confidence intervals (95\% CI) were estimated from 1000 bootstrap replicates

\begin{tabular}{|c|c|c|c|}
\hline & \multicolumn{3}{|c|}{ Day-to-day change in } \\
\hline & $\begin{array}{l}\text { Void volume } \\
\text { (mL) }\end{array}$ & $\begin{array}{l}\text { Intervoid } \\
\text { time }(\mathrm{h})\end{array}$ & $\begin{array}{l}\text { Urine production } \\
\text { rate }(\mathrm{mL} / \mathrm{h})\end{array}$ \\
\hline mean $( \pm \mathrm{SD})$ & $1 \pm 204$ & $0.0 \pm 1.9$ & $\begin{array}{lll}0 & \pm & 27\end{array}$ \\
\hline median $[95 \% \mathrm{CI}]$ & $0.0[-30,0]$ & $-0.1[-0.3,0.0]$ & $0.1[-1.5,2.0]$ \\
\hline skewness $^{*}[95 \% \mathrm{CI}]$ & $0.0[-0.1,0.2]$ & $0.1[-0.4,0.5]$ & $-0.2[-0.7,0.2]$ \\
\hline kurtosis excess $^{*}[95 \% \mathrm{CI}]$ & $0.4[0.1,0.9]$ & $2.3[1.5,3.7]$ & $2.1[0.9,4.3]$ \\
\hline range & {$[-645,605]$} & {$[-8.33,7.67]$} & {$[-135.6,115.2]$} \\
\hline
\end{tabular}

\subsection{Inter-subject variation}

To give some idea of the range of inter-subject variation of void volume, intervoid time and urine production rate, the data are summarised in Figure 3 and Table 3 for each of the 24 subjects. The minimum void volume ranged from 50 $\mathrm{mL}$ to $350 \mathrm{~mL}$ (median $=175 \mathrm{~mL}, \mathrm{IQR}=[118,212] \mathrm{mL}$ ) and the maximum ranged from $540 \mathrm{~mL}$ to $980 \mathrm{~mL}$ (median $=750 \mathrm{~mL}, \mathrm{IQR}=[690,810] \mathrm{mL}$ ). The subjects' ranges of void volumes varied from $300 \mathrm{~mL}$ to $885 \mathrm{~mL}$ around a median of $563 \mathrm{~mL}(\mathrm{IQR}=[498,625] \mathrm{mL})$. This means that $25 \%$ of subjects had at least one pair of void volumes in a cycle that differed by more than $625 \mathrm{~mL}$. However, two subjects ( 3 and 17, Figure 3A) had a range of void volumes of at least 880 $\mathrm{mL}$ and one subject (13, Figure $3 \mathrm{~A}$ ) had a range of void volumes of only $300 \mathrm{~mL}$. Overall, 20 of 24 subjects had at least one void volume in excess of $600 \mathrm{~mL}$ and 17 of 24 subjects had at least one void volume of no more than $200 \mathrm{~mL}$ (Figure $3 \mathrm{~A})$. There was no obvious similarity in the distributions of the void volumes and the intervoid times of the subjects (Figure 3, A and B), consistent with the poor correlation between these variables reported above.

The minimum urine production rate ranged from $10 \mathrm{~mL} \mathrm{~h}^{-1}$ to $44 \mathrm{~mL} \mathrm{~h}^{-1}$ (median $\left.=20 \mathrm{~mL} \mathrm{~h}^{-1}, \mathrm{IQR}=[16,24] \mathrm{mL} \mathrm{h}^{-1}\right)$ and the maximum ranged from 64 $\mathrm{mL} \mathrm{h}^{-1}$ to $187 \mathrm{~mL} \mathrm{~h}^{-1}$ (median $=95 \mathrm{~mL} \mathrm{~h}^{-1}$, IQR $\left.=[77,105] \mathrm{mL} \mathrm{h}^{-1}\right)$. The subjects' ranges of urine production rate varied from $40 \mathrm{~mL} \mathrm{~h}^{-1}$ to $142 \mathrm{~mL} \mathrm{~h}^{-1}$ around a median of $71 \mathrm{~mL} \mathrm{~h}^{-1}\left(\mathrm{IQR}=[57,87] \mathrm{mL} \mathrm{h}^{-1}\right)$. This means that $25 \%$ of subjects 

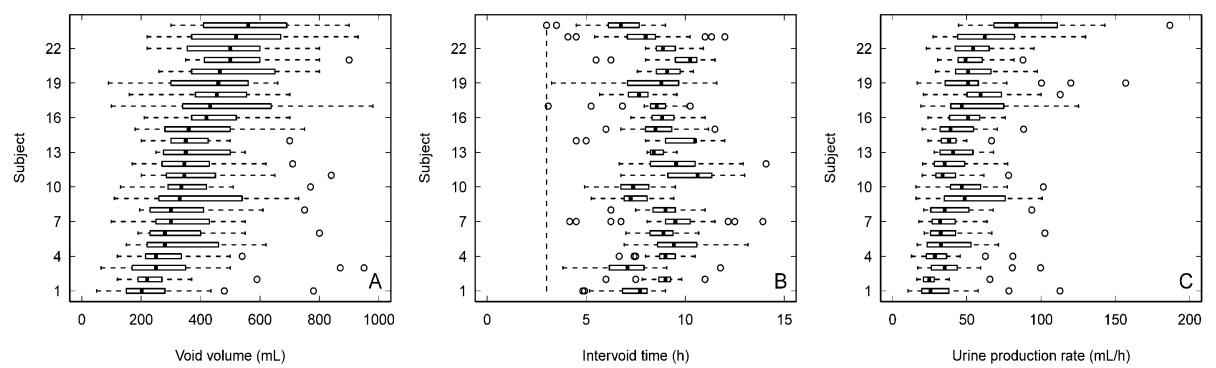

Figure 3. The variability of void volume (A), intervoid time (B) and urine production rate $(\mathrm{C})$ for 24 different women arbitrarily sorted by median void volume. In (B) the vertical dashed line indicates $3 \mathrm{~h}$, the minimum acceptable intervoid time (Brown, 2011). Details of the minima, maxima and ranges are given in Table 3

Table 3. Summary of the properties of the distributions data for individual subjects

(Figure 3), based on $n=653$ samples from 24 women

\begin{tabular}{lccc}
\hline Median & Range of & & \\
\cline { 2 - 4 } & $\begin{array}{c}\text { Void volume } \\
(\mathrm{mL})\end{array}$ & $\begin{array}{c}\text { Intervoid } \\
\text { time }(\mathrm{h})\end{array}$ & $\begin{array}{c}\text { Urine production } \\
\text { rate }(\mathrm{mL} / \mathrm{h})\end{array}$ \\
\hline minimum [range] & $175[50,350]$ & $5.8[3.0,8.1]$ & $20[10,44]$ \\
maximum [range] & $750[540,980]$ & $11.0[9.0,14.1]$ & $95[64,187]$ \\
range [range] & $563[300,885]$ & $5.3[1.5,9.8]$ & $71[40,142]$ \\
\hline
\end{tabular}

had at least one pair of void volumes in a cycle that differed by more than $87 \mathrm{~mL}$ $\mathrm{h}^{-1}$. Overall, 12 subjects had at least one day with a urine production rate of no more than $20 \mathrm{~mL} \mathrm{~h}^{-1}$ and 9 subjects had at least one day with a urine production rate of at least $100 \mathrm{~mL} \mathrm{~h}^{-1}$ (Figure 3C).

\section{Discussion and conclusions}

We have shown that the distributions of void volume and urine production rate are approximately lognormal (Figure 1, A, B, E and F) and the values vary over substantial ranges (0.14-2.6 and 0.24-4.4 times the median for void volume and urine production rate respectively; Table 1). The day-to-day changes in void volume and urine production rate are approximately normally distributed (Figure 2, A, B, E and F) as is expected (Dudewicz and Mishra, 1988 p. 267; Lo, 2012). However, the kurtosis excess is significantly greater than zero in each case (Table 2) from which we infer that the probability of extreme values is greater than would ordinarily be expected for a normally distributed variable. The intervoid 
times are approximately normally distributed (Figure 1, C and D, Table 1), consistent with reports of sleep duration with much larger sample sizes (Hublin et al., 2001; Groeger et al., 2004; Ursin et al., 2005). We have also shown that there is no significant correlation between the void volume and the intervoid time or between the urine production rate and the intervoid time (Figure 3). Finally, we have shown that there can be considerable variation in void volume, intervoid time and urine production rate within just one menstrual cycle and that this varies significantly between women (Figure 3 ).

The approximately lognormal distributions of the void volume and urine production rate (Figure 1, A, B, E and F) are significant in at least three respects. First, they imply that extreme values of these variables, both large and small, are more likely than one would expect for a normally distributed variable. This is also reflected in the distribution of the day-to-day changes in void volume (Figure 2, A, B, E and F, Table 2). Second, they imply that the inclusion of more samples is relatively likely to yield values of these variables that are outside the ranges reported in Table 1. Third, as the quantity of a urinary analyte in a void $(q)$ depends on the excretion rate and the intervoid time and the void volume $(V)$ depends on the urine production rate and the intervoid time, the analyte concentration $(=q / V)$ is a potentially complex variable (Brown et al., 2018). However, considering only the variation in the void volume, for a given $q$, the analyte concentration could vary about 20 -fold (Appendix). A similar argument applies to the urine production rate (Appendix).

The lognormal distribution of the urine volume also largely justifies the assumption that the post-void residual volume (PVR), the volume of urine retained in the bladder after a void, can usually be assumed to be negligible in the assay of urine analytes (Brown et al., 2018). Specifically, if the upper limit of the PVR of a healthy woman is $50 \mathrm{~mL}$ (Al Afraa et al., 2012), then the upper $75 \%$ of void volumes reported here were 5-19.6 times this volume and only $2.5 \%$ of samples had a PVR $\leq 2.6$ times this volume (Figure 1, A and B), although we did observe one void volume of $50 \mathrm{~mL}$ (Table 1). Moreover, the PVR is also asymmetrically distributed, so smaller volumes are more likely than larger volumes, and the reported maxima tend to be much less than $50 \mathrm{~mL}$ (Kolman et al., 1999; Pfisterer et al., 2007; Barapatre et al., 2009). 
We have identified the developing tendency to consider unnecessary any correction for urine production rate in the case of measurements of E1G and PdG in monitoring the menstrual cycle (Miro et al., 2004; MacGregor et al., 2006; Ziomkiewicz et al., 2012; Ecochard et al., 2013; Johnson et al., 2015). There are other examples of such an approach (Murakami and Kawakami, 1992; Charlton et al., 2014), but these relate to situations where very large-scale spot sampling is contemplated. The requirements of users of such assays are rather different from those of a woman monitoring her own fertility every day. In other cases, such as the monitoring of heavy metal exposure by urinalysis, not only is correction advocated, but it is demonstrated that the best means of achieving this is to correct using the urine production rate (Araki et al., 1986a; Araki et al., 1986b; Weaver et al., 2014). We have shown previously that urine production rate correction of E1G concentrations is necessary if the intention is to obtain an estimate of the corresponding serum analyte concentration (Brown et al., 2018).

Based on the data shown in Figure 1B it is possible to estimate the probability of observing a particular relative concentration as a result of failing to account for variations in void volume (Figure 4A). Any comparison of urinary analyte concentrations implies an assumption about the void volume that may or may not be reasonable. For example, each of two urine samples that happen to contain the same quantity of an analyte has a concentration that depends on the void volume. If one void volume is $V_{0}=370 \mathrm{~mL}$, the median void volume we observed (Table 1 ), and the corresponding analyte concentration is $c_{0}$, the probability that the analyte concentration of the other sample $(c)$ is less than or equal to $0.5 c_{0}$ is 0.059 and that of $c \geq 2 c_{0}$ is 0.072 just because of the variability of the void volume (we use 0.5 and 2 for convenience; much larger and smaller multipliers are possible, as is apparent from Figure 4). However, if $V_{0}=750 \mathrm{~mL}$, which is the median maximum void volume observed in a cycle (Table 3 ), these probabilities are quite different: $P\left(c \leq 0.5 c_{0}\right)<0.001$ and $P\left(c \geq 2 c_{0}\right)=0.533$ (Figure 4A). On the other hand, if $V_{0}=175 \mathrm{~mL}$, the median minimum void volume observed in a cycle (Table 3), these probabilities are essentially reversed: $P\left(c \leq 0.5 c_{0}\right)=0.527$ and $P\left(c \geq 2 c_{0}\right)<0.001$ (Figure 4A). In summary, (a) the more $V_{0}$ differs from the median void volume, the more likely it is that $c$ and $c_{0}$ will differ just because of differences in the void volume; and (b) if $V_{0}$ is larger or smaller than the median 
void volume it is more likely that $c>c_{0}$ or $c<c_{0}$, respectively. An analogous argument can be made using the urine production rate and analyte excretion rate instead of void volume and analyte quantity, respectively (Figure 4B).

Three factors should be noted in connection with these calculations. First, we have been conservative in using the median minima and maxima because the actual ranges of variation in void volume (the grey region in Figure 4A) and urine production rate (the grey region in Figure 4B) are rather greater (Table 1). Second, the likelihood of extreme values is much greater if the urine production rate (Figure 4B) rather than just the void volume (Figure 4A) is considered, as would be expected given that the former includes both the latter and the variation in the intervoid time. We infer from this that the extreme values of the urine production rate could lead to greater $c / c_{0}$ than those of the void volume extrema. Third, given the approximately lognormal distribution of the void volume and the urine production rate, it is likely that as more samples are considered the range will also increase. This is consistent with the data of Hays et al. (2015), who reported urine production rates of less than $1 \mathrm{~mL} \mathrm{~h}^{-1}$ and greater than $1000 \mathrm{~mL} \mathrm{~h}$ ${ }^{1}$ among a sample of 14,631 participants, compared with a range of $10-187 \mathrm{~mL}$ $\mathrm{h}^{-1}$ for the 24 women in our data. Of course, their subjects were much more heterogeneous (both male and female, children to elderly, providing mostly daytime urine samples) and they were not excluded on the basis of ill-health, but this is an indication of the sort of values that might be encountered in daily measurements of E1G and PdG in thousands of women (Brown et al., 2017).

It has been reported that the $24 \mathrm{~h}$ urine production rate differs between the follicular and luteal phases of the cycle (Fong and Kretsch, 1993) and fluid retention also differs between the phases (White et al., 2011). Given the number of samples available to us, we have not investigated this possibility. However, such a systematic variation in urine production rate might lead one to expect that the smoothing approach described by Miro et al. (2004) is likely to be biased by the phase of the cycle, although the extent of this is not clear. 

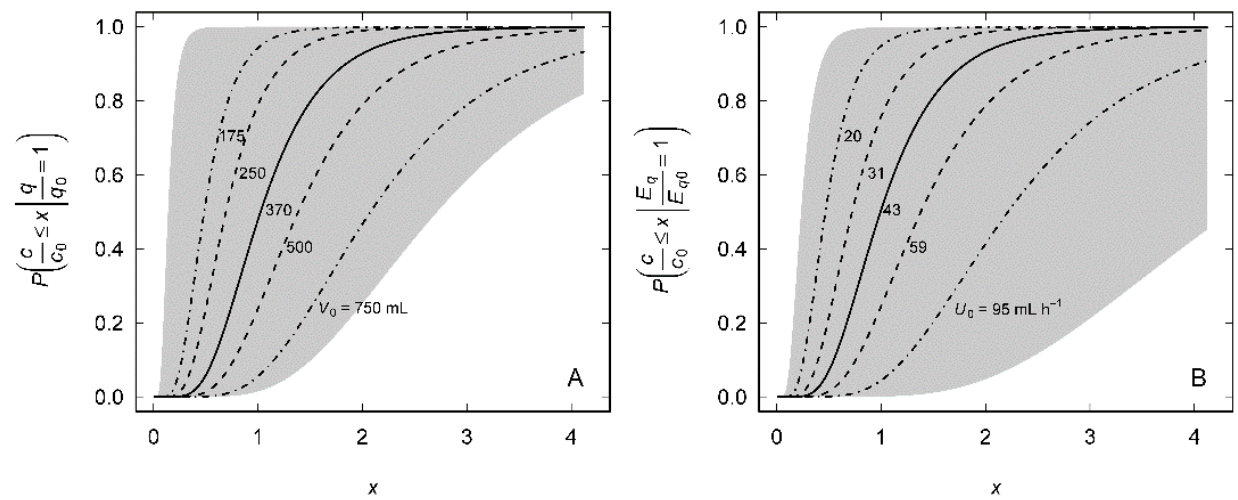

Figure 4. Conditional probability of observing a relative analyte concentration $\left(c / c_{0}\right)$ less than or equal to $x$ due to changes in void volume alone (A) or urine production rate (B). As outlined in the Appendix, the curves are based on the fits to the data shown in Figures $1 \mathrm{~B}(\mathrm{~A})$ and $1 \mathrm{~F}(\mathrm{~B})$. The assumed values of $V_{0}$ and $U_{0}$ were the median (-), lower and upper quartiles (-- $)$, and the medians of the subject minima and maxima (-

.-- ) . The grey zones represent the ranges of void volume $(50-980 \mathrm{~mL})$ and urine production rate $\left(10-187 \mathrm{~mL} \mathrm{~h}^{-1}\right)$ in the data (Table 1)

The main weakness of our analysis is that data from overnight samples from a single cycle of each of only 24 women have been considered. This precludes any consideration of intra-subject variation and constrains analysis of the likelihood of extreme values. We will address some of these issues in due course.

\section{APPENDIX}

Consider a urine sample of volume $V$ and intervoid time $\Delta t$ that contains an analyte at concentration $c$. The quantity of analyte $(q)$ accumulated in the urine during $\Delta t$ is $c V$ and the excretion rate $\left(E_{q}\right)$ is $c V / \Delta t$, where $V / \Delta t$ is the urine production rate $(U)$. Based on Figure $1 \mathrm{~A}, V$ can vary from $50 \mathrm{~mL}$ to $980 \mathrm{~mL}$ (Table 1), with a probability that can be estimated from Figure 1B, so it might be argued that $c=q / V$ could range from $q / 980$ to $q / 50$, which is 19.6 times the minimum concentration, without any change in $\Delta t, E_{q}$ or $q$. A similar result is obtained if it is assumed that $E_{q}$ (rather than $q$ ) is constant, in which case $U$ could vary from about $10 \mathrm{~mL} \mathrm{~h}^{-1}$ to about $187 \mathrm{~mL} \mathrm{~h}^{-1}$ (Table 1), with a probability that can be estimated from Figure $1 \mathrm{~F}$, so $c=E_{q} / U$ could range from $E_{q} / 187$ to $E_{q} / 10$, 
which is 18.7 times the minimum concentration. As we have suggested in the text, a larger sample size is likely to yield a considerable increase in this range (Hays et al., 2015).

As both $U$ and $V$ are approximately lognormally distributed (Figure 1, B and F), the probability density function is

$$
f(x ; \mu, \sigma)=\frac{1}{x \sigma \sqrt{2 \pi}} \exp \left(-\frac{1}{2}\left(\frac{\ln (x)-\mu}{\sigma}\right)^{2}\right),
$$

where $x$ is either $U$ or $V$ as required and $\mu$ and $\sigma$ are the mean and standard deviation, respectively, of $\ln (x)$. The corresponding cumulative density function $(\mathrm{CDF})$ is

$$
F(x ; \mu, \sigma)=P(X \leq x)=\frac{1}{2}+\frac{1}{2} \operatorname{erf}\left(\frac{\ln (x)-\mu}{\sqrt{2} \sigma}\right),
$$

where $\operatorname{erf}(\cdot)$ is the error function, which is the smooth curve shown in each of Figures $1 \mathrm{~B}$ and $1 \mathrm{~F}$.

To estimate the probability of observing a particular analyte concentration due to a change in only the void volume, as is shown in Figure 4A, we assume that two urine samples contain the same quantity of analyte $\left(q=q_{0}\right)$. However, one sample has specified void volume $\left(V_{0}\right)$ and analyte concentration $\left(c_{0}\right)$ and the other has void volume $(V)$ and the corresponding analyte concentration $(c)$, so $q$ $=c V=c_{0} V_{0}$ and $c / c_{0}=V_{0} / V$. It follows from this that, if both samples contain the same quantity of analyte $\left(q=q_{0}\right)$, the probability of observing $c / c_{0} \leq x$ because of the natural variation in $V$ is

$$
P\left(\frac{c}{c_{0}} \leq x \mid \frac{q}{q_{0}}=1\right)=P\left(\frac{V_{0}}{V} \leq x\right) .
$$

As $V$ is approximately lognormally distributed (Figure $1 \mathrm{~B}$ ) and the CDF is $F(x ; \mu, \sigma), 1 / V$ is also lognormally distributed, but the $\mathrm{CDF}$ is $F(x ;-\mu, \sigma)$, so the CDF of $V_{0} / V$ is $F\left(x ;-\mu+\ln \left(V_{0}\right), \sigma\right)$.

Very similar reasoning leads to an expression based on $U$ (Figure 4B). In this case, we assume that the two urine samples have the same excretion rate $\left(E_{q}=\right.$ 
$\left.E_{q 0}\right)$. However, one sample has specified urine production rate $\left(U_{0}\right)$ and analyte concentration $\left(c_{0}\right)$ and the other has urine production rate $(U)$ and the corresponding analyte concentration $(c)$, so $E_{q}=E_{q 0}=c U=c_{0} U_{0}$ and $c / c_{0}=U_{0} / U$. If both samples have the same excretion rate $\left(E_{q}=E_{q 0}\right)$, the probability of observing $c / c_{0} \leq x$ because of the natural variation in $U$ is

$$
P\left(\frac{c}{c_{0}} \leq x \mid \frac{E_{q}}{E_{q 0}}=1\right)=P\left(\frac{U_{0}}{U} \leq x\right) .
$$

As for $V, U$ is approximately lognormally distributed (Figure $1 \mathrm{~F}$ ) and it follows that the $\mathrm{CDF}$ of $U_{0} / U$ is $F\left(x ;-\mu+\ln \left(U_{0}\right), \sigma\right)$.

\section{REFERENCES}

Al Afraa T., Mahfouz W., Campeau L., Corcus J. (2012): Normal lower urinary tract assessment in women: I. Uroflowmetry and post-void residual, pad tests, and bladder diaries. Int. Urogynecol. J. 23: 681-685.

Araki S., Aono H., Murata K. (1986a): Adjustment of urinary concentration to urinary volume in relation to erythrocyte and plasma concentrations: an evaluation of urinary heavy metals and organic substances. Arch. Environ. Health 41: 171-177.

Araki S., Murata K., Aono H., Yanagihara S., Niinuma Y., Yamamoto R., Ishihara N. (1986b): Comparison of the effects of urinary flow on adjusted and nonadjusted excretion of heavy metals and organic substances in 'healthy' men. J. Appl. Toxicol. 6: 245-251.

Barapatre Y., Agarwal M.M., Singh S.K., Sharma S.K., Mavuduru R., Mete U.K., Kumar S., Mandal A.K. (2009): Uroflowmetry in healthy women: development and validation of flow-volume and corrected flow-age nomograms. Neurourol. Urodynamics 28: 1003-1009.

Barr D.B., Wilder L.C., Caudill S.P., Gonzalez A.J., Needham L.L., Pirkle J.L. (2005): Urinary creatinine concentrations in the U. S. population: implications for urinary biologic monitoring measurements. Environ. Health Perspectives 113: 192-200.

Blackwell L.F., Brown J.B., Vigil P., Gross B., Sufi S., d'Arcangues C. (2003): Hormonal monitoring of ovarian activity using the Ovarian Monitor, part I. Validation of home and laboratory results obtained during ovulatory cycles by comparison with radioimmunoassay. Steroids 68: 465-476.

Blackwell L.F., Vigil P., Alliende M.E., Brown S., Festin M., Cooke D.G. (2016): Monitoring of ovarian activity by measurement of urinary excretion rates using the Ovarian Monitor, part IV: the relationship of the pregnanediol glucuronide threshold, basal body temperature and cervical mucus as markers for the beginning of the postovulatory infertile period. Human Reprod. 31: 445-453.

Blackwell L.F., Vigil P., Cooke D.G., d'Arcangues C., Brown J.B. (2013): Monitoring of ovarian activity by daily measurement of urinary excretion rates of estrone 
glucuronide and pregnandediol glucuronide using the Ovarian Monitor, part III: Variability of normal menstrual cycle profiles. Human Reprod. 28: 3306-3315.

Blackwell L.F., Vigil P., Gross B., d'Arcangues C., Cooke D.G., Brown J.B. (2012): Monitoring of ovarian activity by measurement of urinary excretion rates of estrone glucuronide and pregnanediol glucuronide using the ovarian monitor, part II: reliability of home testing. Human Reprod. 27: 550-557.

Brown J.B. (2011): Types of ovarian activity in women and their significance: the continuum (a reinterpretation of early findings). Human Reprod. Update 17: 141-158.

Brown J.B., Blackwell L.F., Holmes J., Smyth K. (1989): New assays for identifying the fertile period. Int. J. Gynecol. Obstet. suppl 1: 111-22.

Brown S. (2017): The distribution of phoneme inventory and language evolution. Cultural Anthropol. Ethnosemiotics 3: 22-34.

Brown S., Blackwell L.F., Cooke D.G. (2017): Online fertility monitoring: some of the issues. Int. J. Open Information Technol. 5: 85-91.

Brown S., Cooke D.G., Blackwell L.F. (2018): Monitoring the menstrual cycle using urine oestrone glucuronide: the relationship between excretion rate and concentration. Int. J. Basic Med. Sci. Pharm., in press.

Charlton K.E., Batterham M.J., Buchanan L.M., Mackerras D. (2014): Intraindividual variation in urinary iodine concentrations: effect of adjustment on population distribution using two and three repeated spot urine collections. BMJ Open 4: e003799.

Cooke D.G. (2000). Homogeneous and heterogeneous enzyme immunoassays for the home detection of fertility. PhD thesis, Massey University.

Côté A.-M., Firoz T., Mattman A. (2008): The 24-hour urine collection: gold standard of historical practice? Am. J. Obstet. Gynecol. 199: 625.e1-625.e6.

Craig A., Cloarec O., Holmes E., Nicholson J.K., Lindon J.C. (2006): Scaling and normalization effects in NMR spectroscopic metabonomic data sets. Anal. Chem. 78: 2262-2267.

Dieterle F., Ross A., Schlotterbeck G., Senn H. (2006): Probabilistic quotient normalization as robust method to account for dilution of complex biological mixtures. Application in ${ }^{1} \mathrm{H}$ NMR metabonomics. Anal. Chem. 78: 4281-4290.

Dudewicz E.J., Mishra S.N. (1988): Modern mathematical statistics. John Wiley and Sons, Inc., New York.

Ecochard R., Leiva R., Bouchard T., Boehringer H., Direito A., Mariani A., Fehring R. (2013): Use of urinary pregnanediol 3-glucuronide to confirm ovulation. Steroids 78: 1035-1040.

Fong A.K.H., Kretsch M.J. (1993): Changes in dietary intake, urinary nitrogen, and urinary volume across the menstrual cycle. Am. J. Clin. Nutr. 57: 43-46.

Gaspari F., Perico N., Remuzzi G. (2006): Timed urine collections are not needed to measure urine protein excretion in clinical practice. Am. J. Kidney Diseases 47: 1-7.

Groeger J.A., Zijlstra F.R.H., Dijk D.-J. (2004): Sleep quantity, sleep difficulties and their perceived consequences in a representative sample of some 2000 British adults. J. Sleep Res. 13: 359-371.

Hays S.M., Aylward L.L., Blount B.C. (2015): Variation in urinary flow rates according to demographic characteristics and body mass index in NHANES: potential 
confounding of associations between health outcomes and urinary biomarker concentrations. Environ. Health Perspectives 123: 293-300.

Hublin C., Kaprio J., Partinen M., Koskenvuc M. (2001): Insufficient sleep - a population-based study in adults. Sleep 24: 392-400.

Ihaka R., Gentleman R. (1996): R: a language for data analysis and graphics. J. Comput. Graphical Stat. 5: 299-314.

Ji C., Sykes L., Paul C., Dary O., Legetic B., Campbell N.R.C., Cappuccio D.P. (2012): Systematic review of studies comparing 24-hour and spot urine collections for estimating population salt intake. Rev. Panamericana Salud Publica 32: 307-315.

Johnson S., Weddell S., Godbert S., Freundl G., Roos J., Gnoth C. (2015): Development of the first urinary reproductive hormone ranges referenced to independently determined ovulation day. Clin. Chem. Lab. Med. 53: 1099-1108.

Kolman C., Girman C.J., Jacobsen S.J., Lieber M.M. (1999): Distribution of post-void residual urine volume in randomly selected men. J. Urol. 161: 122-127.

Lane C., Brown M., Dunsmuir W., Kelly J., Mangos G. (2006): Can spot urine protein/creatinine ratio replace $24 \mathrm{~h}$ urine protein in usual clinical nephrology? Nephrol. 11: 245-249.

Lo C.F. (2012): The sum and difference of two lognormal random variables. J. Appl. Math. 2012: 838397.

MacGregor E.A., Frith A., Ellis J., Aspinall L., Hackshaw A. (2006): Incidence of migraine relative to menstrual cycle phases of rising and falling estrogen. Neurol. 67: 2154-2158.

Miller M., Simundic A.-M. (2013): Low level of adherence to instructions for 24-hour urine collection among hospital outpatients. Biochemia Medica 23: 316-320.

Miro F., Coley J., Gani M.M., Perry P.W., Talbot D., Aspinall L.J. (2004): Comparison between creatinine and pregnanediol adjustments in the retrospective analysis of urinary hormone profiles during the human menstrual cycle. Clin. Chem. Lab. Med. 42: 1043-1050.

Murakami T., Kawakami H. (1992): Urine concentration adjustment with a dipstick is dispensable for urinary $\beta_{2}$-microglobulin screening in children. Japanese J. Nephrol. 34: 29-32.

Pearson K. (1897): Mathematical contributions to the theory of evolution. On a form of spurious correlation which may arise when indices are used in the measurement of organs. Proc. R. Soc. Lond. 60: 489-498.

Perrone R.D., Madias N.E., Levey A.S. (1992): Serum creatinine as an index of renal function: new insights into old concepts. Clin. Chem. 38: 1933-1953.

Pfisterer M.H.-D., Griffiths D.J., Rosenberg L., Schaefer W., Resnick N.M. (2007): Parameters of bladder function in pre-, peri-, and postmenopausal continent women without detrusor overactivity. Neurourol. Urodynamics 26: 356-361.

Rasmussen L.G., Savorani F., Larsen T.M., Dragsted L.O., Astrup A., Engelsen S.B. (2011): Standardization of factors that influence human urine metabolomics. Metabolomics 7: 71-83.

Remer T., Neubert A., Maser-Gluth C. (2002): Anthropometry-based reference values for 24-h urinary creatinine excretion during growth and their use in endocrine and nutritional research. Am. J. Clin. Nutr. 75: 561-569. 
Shidham G., Herbert L.A. (2006): Timed urine collections are not needed to measure urine protein excretion in clinical practice. Am. J. Kidney Diseases 47: 8-14.

Thudichum J.L.W. (1858): A treatise on the pathology of the urine, including a complete guide to its analysis. John Churchill, London.

Ursin R., Bjorvatn B., Holsten F. (2005): Sleep duration, subjective sleep need, and sleep habits of 40- to 45-year-olds in the Hordaland Health study. Sleep 28: 1260-1269.

Weaver V.M., Vargas G.G., Silbergeld E.K., Rothenberg S.J., Fadrowski J.J., RubioAndrade M., Parsons P.J., Steuerwald A.J., Navas-Acien A., Guallar E. (2014): Impact of urine concentration adjustment method on associations between urine metals and estimated glomerular filtration rates (eGFR) in adolescents. Environ. Res. 132: 226-232.

White C.P., Hitchcock C.L., Vigna Y.M., Prior J.C. (2011): Fluid retention over the menstrual cycle: 1-year data from the Prospective Ovulation Cohort. Obstet. Gynecol. Int. 2011: 138451.

Ziomkiewicz A., Wichary S., Bochenek D., Pawlowski B., Jasienska G. (2012): Temperament and ovarian reproductive hormones in women: evidence from a study during the entire menstrual cycle. Hormones Behavior 61: 535-540. 